\title{
A Novel Thyroid Hormone Mediated Regulation of HSV-1 Gene Expression and Replication is Specific to Neuronal Cells and Associated with Disruption of Chromatin Condensation
}

\author{
Feng Chen, Jay Palem, Matthew Balish, Robert Figliozzi, Amakoe Ajavon, and S \\ Victor Hsia*
}

Department of Pharmaceutical Sciences, School of Pharmacy, University of Maryland Eastern Shore, Princess Anne, MD 21853 Maryland, United States

Received: December 19, 2013; Accepted: February 06, 2014; Published: February 07, 2014

*Corresponding author: S Victor Hsia, Department of Pharmaceutical Sciences, School of Pharmacy, University of Maryland Eastern Shore, Princess Anne, MD 21853 Maryland, United States, Tel: 410-621-2240; Fax: 410-651-8394; E-mail: vhsia@umes.edu

\begin{abstract}
In earlier studies, we have shown that thyroid hormone (T3) regulated the Herpes Simplex Virus Type -1 (HSV-1) gene expression and replication through its nuclear receptor TR via histone modification and chromatin remodeling in a neuroblastoma cell line neuro-2a cells (N2a). This observation suggested that T3 regulation may be neuron-specific and it may have implication in HSV-1 latency and reactivation. The present study conducted on in-vitro demonstrated that removal of T3 can de-repress the HSV-1 replication and favor reactivation. Transfection studies and infection assays indicated that HSV-1 thymidine kinase (TK), a key viral gene during reactivation, was repressed by TR/T3 in cells with neuronal origin but not in non-neuronal cells. Additional studies showed that RCC1 (Regulator of Chromosome Condensation 1) was sequestered but efficiently detected upon viral infection in N2a cells. Western blot analyses indicated that addition of T3 repressed the RCC1 expression upon infection. It is likely that diminution of RCC1 upon infection in neuronal cells under the influence of TR/T3 may lead to repression of viral replication/gene expression, thus promoting latency. Together these results demonstrated that TR/T3 mediated regulation is specific to neuronal cells and differential chromosome condensation may play a critical role in this process.
\end{abstract}

Keywords: HSV-1; Thyroid hormone; Chromosome; Neuron; Latency

\section{Abbreviations}

MOI: Multiplicity of Infection; hpi: Hours Post Infection; dpi: Days Post Infection

\section{Introduction}

HSV-1 is responsible for a number of infectious complications in humans (Lopez Garcia, Enriquez [1-2]). After a brief period of primary acute infection, the virus may launch a dormant infection in the sensory neuron of trigeminal ganglia for life and reactivation occurs over the different areas of lips collectively called cold sores or fever blisters [3]. HSV-1 can often lead to other clinical symptoms at different sites such as cornea (herpes keratitis) [4], finger and nail (herpetic whitlow)[5], skin (herpes dermatitis) [6], etc. The virus may result in lethal complications, for example, herpes encephalitis. Approximately 10\% of viral encephalitis derived from HSV-1 [7] and reactivation from the latency in the trigeminal ganglia seemed to be playing a critical role although the molecular mechanisms leading to HSV encephalitis remained vague [8].

Various mechanisms have been suggested to be involved in the establishment of HSV-1 latency and momentary reactivation [9]. Of all these mechanisms, hormonal regulation/imbalance was discussed but not extensively studied [10-13]. For example, it was shown that thyroid hormone $(\mathrm{TH})$ plays a role in regulating HSV-1 key genes and also participates in latency/reactivation; but detailed molecular pathways involved in regulating these mechanisms are still unclear [14-16].

TH is produced from the thyroid gland and there are two products, thyroxine (T4) and triiodothyronine (T3), and the latter is more potent and responsible for the majority of biological effects [17]. T3 employs its function via its nuclear receptors (TRs), which are transcription factors usually dimerized with other nuclear receptors [18]. Two major TR isoforms were characterized. TR- $\alpha 1$ is broadly expressed with high level of expression in cardiac and skeletal muscles. TR- $\beta 1$, however, is predominately produced in brain, liver and kidney [19]. It has been shown that both liganded and unliganded TRs are involved in gene regulation.

In addition to $\mathrm{TH}$ effects, roles of repressive chromatin/ chromosome condensation on latent HSV-1 genome raised significant interest during the past decade [20-26]. The present investigation focused on the interaction of T3, TRs, and chromosome condensation during HSV-1 gene regulation in neuronal cells. The gene regulation of thymidine kinase (TK) 
was analyzed in neuroblastoma cells $\mathrm{N} 2 \mathrm{a}$ and $\mathrm{N} 2 \mathrm{aTR} \beta$ (N2a cell constitutively expressing TR $\beta$ ). Our results indicated that T3/TR regulated the TK promoter activity and this regulatory effect is neuronal-specific with participation of chromosome condensation.

\section{Methods}

\section{Viruses, cell lines, and culture conditions}

The neuroblastoma cell line N2a (mouse; ATCC ${ }^{\circledR}$ CCL-131 ${ }^{\mathrm{m}}$ ) and SK-N-MC (human; ATCC ${ }^{\circledR}$ HTB-10 ${ }^{\mathrm{mm}}$ ) were obtained from the American Type Culture Collection (ATCC) and cultured in DMEM/F12 medium supplemented with $10 \%$ charcoal-treated fetal bovine serum (FBS).

N2aTR $\beta$ cell was a generous gift from Dr. Robert Denver (University of Michigan, Ann Arbor, MI) and was maintained under the same conditions of N2a. Vero cells and HEK293 were cultured in $10 \%$ FBS supplemented with DMEM according to ATCC. All cells were cultured at $37^{\circ} \mathrm{C}$ with $5 \% \mathrm{CO}_{2}$. HSV- 1 strain $17 \mathrm{Syn}^{+}$-EGFP was a gift from Dr. Gus Kousoulas at the Louisiana State University [27]. T3 was acquired from Sigma (St. Louis, MO).

\section{Plasmids and introduction of point mutation}

The pRL-TK vector was procured from Promega (Madison, WI; Cat\#: TB240) and it contains HSV-1 TK promoter driving luciferase as reporter. Transfection efficiency control was achieved by using Monster Green ${ }^{\circledR}$ Fluorescent Protein phMGFP Vector (Promega, Cat\#: E6421). Normalization was accomplished by using plasmid pICP4-SEAP containing reporter secreted alkaline phosphatase (SEAP) driven by ICP4 promoter [25]. Sitedirected mutagenesis at the TK TRE was essentially described previously [15].

\section{Transfection}

For non-neuronal cells, plasmids were transfected using lipofectame 2000 and DNA was mixed in Opti- MEM medium to allow complex formation according to the manufacturer (Life Technology, CA). N2a and N2aTR $\beta$ cells were transfected using Nucleofector II from Amaxa

(Cat\#: AAD-1001S, Gaithersburg, MD) for high efficiency of transfection (protocol essentially described by the manufacturer) using Kit V (Amaxa.Cat\#: VCA-1005) and the protocol number was T-024. Plasmid phMGFP was cotransfected as a control.

\section{Reporter assays}

Transfected cells were harvested $48 \mathrm{hr}$ after transfection and the protocol was essentially described by the manufacturer (Promega, WI). Promoter activity was analyzed by measuring the luminescence over a 10 -sec interval with a 2-sec delay on the $20 / 20^{\text {n }}$ Luminometer (Turner Biosystem, Sunnyvale, CA). The results were presented as the fold induction of the reporter plasmid in the presence or absence of T3 (100 nM). The SEAP (Secreted Alkaline Phosphtase) assay was used as an internal control [23]. All experiments were performed in triplicates and were repeated twice.

\section{Infection}

The N2a and Vero cells were plated in $35 \mathrm{~mm}$ dish a day before infection. On the day of infection the medium was removed and the cells were washed with PBS and then infected with $250 \mu \mathrm{l}$ of medium containing $1 \mathrm{MOI}$ of virus. At the $1 \mathrm{hpi}$ medium was replaced by fresh medium.

\section{Antibodies}

Anti-RCC1 is a mouse monoclonal antibody recognizing amino acids 122-421 purchased from Santa Cruz Biotechnology (Cat\#: sc-55559) with 1:500 dilution for Western blot analyses. Anti-Ran is a rabbit polyclonal antibody obtained from Abcam (Cat\#: ab4781). Anti-GFP is a rabbit antibody from Sigma-Aldrich (Cat\#: G1544). Anti- $\alpha$ Tubulin antibody was procured from Calbiochem (Cat\#: CP06). It is a mouse monoclonal antibody. All these antibodies used for Western blot analyses or immunofluorescent assays had the dilution factors performed according to the manufacturer's suggestions.

\section{Immuno-fluorescence}

Cells were treated based on the experimental needs a day before the test. To obtain the best results, approximately 20,000 cells/well were placed in a multi-chamber slide (Cat\# 354104 BD Falcon). After infection cells were washed once with $2 \mathrm{ml}$ PBS for 5 min and fixed with $100 \%$ methanol at $-20^{\circ} \mathrm{C}$. Chamber slides were further incubated with $2 \%$ blocking serum followed by incubation with primary antibody overnight at $4^{\circ} \mathrm{C}$. Fluorescent conjugated secondary antibody (Invitrogen cat\# A21424) was used at RT for $1 \mathrm{hr}$. To finish, fluorescent mounting medium containing DAPI was applied for microscopy. The detection of EGFP, red fluorescence, and DAPI staining were recorded by an Olympus fluorescence microscope IX71 equipped with a digital camera photo apparatus DP71.

\section{Western blotting}

Cell extract was subjected to gel electrophoresis and transferred to membrane using iBlot ${ }^{\circledR}$ Gel Transfer Device (Life Technology, Cat\#: IB1001). Anti-RCC1 was added (Cat\#: sc-55559) with 1:500 dilution and 1:1000 for anti-GFP (Cat\#: G1544). Anti- $\alpha$-Tubulin, as an internal control was used at a dilution of 1:10,000. The chemi-luminescent signal was detected by Bio-Rad Chemi-docl XRS imaging systems (Hercules, CA). Our established protocol was described [28].

\section{T3 removal experiments}

N2a and N2aTR $\beta$ cells were prepared approximately 50\% on a poly-D-lysine coated 6-well plate with $100 \mathrm{nM}$ T3. Fresh T3 were refreshed every two days. On day 6, infection was performed at moi of 1 . At 5 days post-inoculation (dpi), the media was collected and cells were washed with $1 \mathrm{ml}$ of PBS followed by the addition of $1 \mathrm{ml}$ of fresh media with or without $\mathrm{T} 3$ for 48 $h$. at the end of the experiments, the media were collected and subjected to plaque assays for replication analyses.

\section{Plaque assays}

Media collected from negative controls and infected cells were subjected to plaque assays by infecting Vero cells. Cell monolayers at $100 \%$ confluency in 24-well plates were incubated with $200 \mu \mathrm{l}$ of media for $45 \mathrm{~min}$ on a rocking platform at different dilutions 
followed by addition of $1 \mathrm{ml}$ fresh media to each well for $48 \mathrm{~h}$. At the end the infected cells were rinsed with PBS and treated with crystal violet (PML Microbiologicals, Wilsonville, OR) for $20 \mathrm{~min}$ followed by washing with water. Plaques were counted in each triplicated well and the probability was measured by a Student's paired $t$-Test with a two-tailed distribution using Microsoft Excel.

\section{Results}

\section{Removal of $\mathrm{T} 3$ reversed the hormone-mediated repression of $\mathrm{HSV}-1$ replication}

The regulatory effect of $\mathrm{T} 3$ on viral replication was tested using our in vitro latency cell culture model. The results showed that on Day 5 while T3 is present, the liganded TR exerted stronger inhibitory effects on the viral replication (Figure 1, compare bar 2, 3 and 7, 8). Subsequent observation indicated that viral replication continued to decrease after $7 \mathrm{dpi}$, particularly in the presence of T3 (Figure 1, compare bar 4 and 9). Fluorescent microscopy detected similar numbers of cells producing green fluorescent signals confirming that the number of infected cells were equivalent (data not shown). Interestingly, the replication increased drastically when the hormone was washed out only when the TR is present (Figure 1, compare bar 4, 5, 9, and 10). These results demonstrated that T3/TR repressed HSV-1 replication in neuronal cells. This inhibition is reversible since hormone washout can restore the replication capability.

\section{Regulation of HSV-1 TK by T3 and TR was specific to neuronal cells}

To test this hypothesis, neuronal cells N2a and nonneuronal cells 293HEK were co-transfected with pRL-TK and TR $\beta$ expression vector pTR $\beta$ in the absence or presence of T3 followed by reporter assays. The Luc assays data revealed a dosedependent repression on TK promoter activity in the presence of T3 only in N2a cells but not in HEK293, which is a non-neuronal, human embryonic kidney cells (Figure 2A and 2B). This hypothesis was further characterized by transfecting a human neuronal cells SK-N-MC and the results confirmed the previous observation that hormone-mediated repression was limited to cells with neuronal origin (Figure 3). It has been suggested that HEK293 exhibited several neuronal makers [29]. In our opinion, this T3/TR mediated regulation is likely due to a complex scenario involving not only neuronal factors but also differentiation condition since HEK293 never displayed an obvious differentiation under standard culture condition. It is unclear why a human embryonic kidney cell line would reveal neuronal makers but continue to proliferate without differentiation, a signature of neurons. We also found that other non-neuronal cells such as Vero showed no T3/TR regulation on HSV-1 TK (unpublished data). N2a cells however, unveiled a differentiation morphology and physiology in the presence of TR $\beta$ and T3 (Lebel JM, et al. [30]). TThese results collectively indicated that neuronal cellular environment as well as differentiation status and specific epigenetic control may contribute to this novel regulation. TK is not required for lytic infection. However, it is essential for viral replication in neurons to generate dNTP since it is in a resting state [15]. Besides, viral replication is critical for efficient $\alpha$ and $\beta$ expressions in neurons during reactivation; in spite of occurring during the later stage of lytic infection [31]. Therefore TK has been proposed to promote $\alpha$ transcription and replication during reactivation [32]. This hypothesis was further supported by in vivo studies that TK was one of the first genes to be produced [33] and TK-null mutant exhibited poor $\alpha$ and $\beta$ expression during reactivation [34].

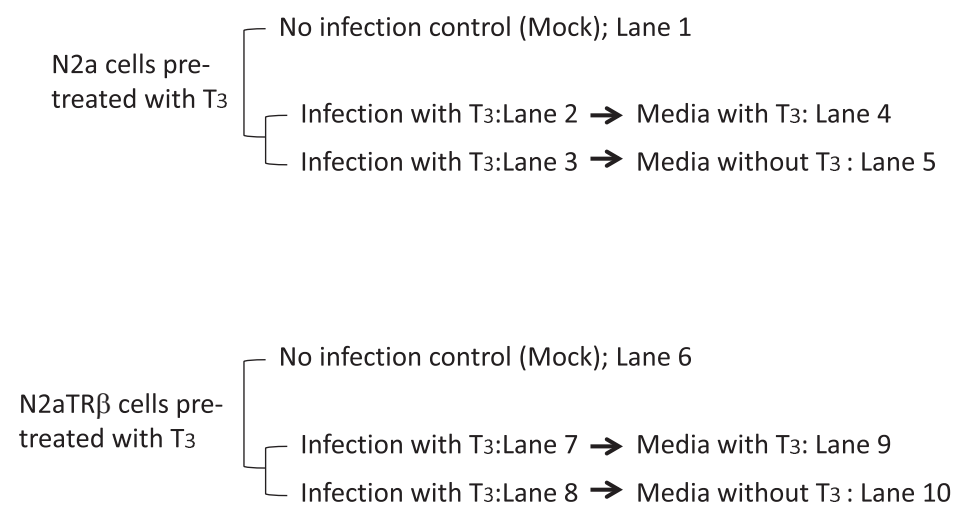

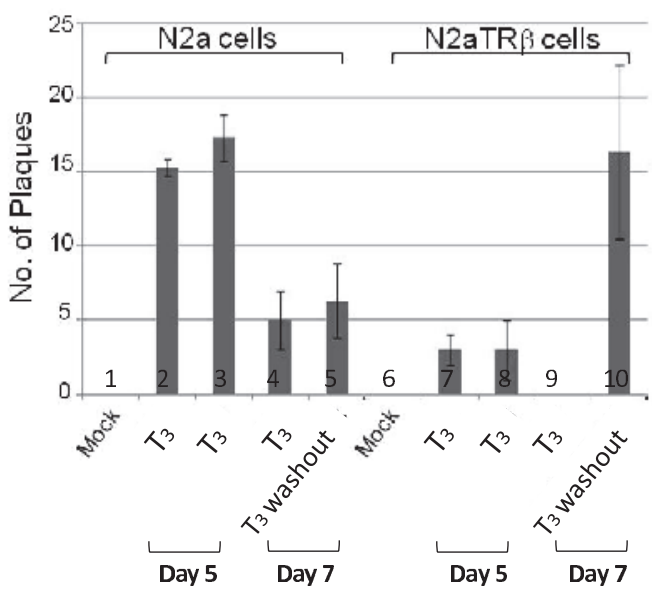

Figure 1: T3 washout increased the release of infectious HSV-1 in N2aTR $\beta$ cells

A. Scheme of infection and T3 treatment.

B. Cells were infected with HSV-1 at moi of 1 in the presence of T3. At Day 5 post-infection the media were removed/collected and fresh media with or without T3 were added. At Day 7 post-infection the media were collected and subjected to plaque assays. Lane 1 and 6: no infection control; Lane $2,3,7$, and 8: infection with thyroid hormone T3 and the plaque assays using media collected on Day 5; Lane 4 and 9: plaque assays performed using media collected on wells from Lane 2 and 7, respectively; Lane 5 and 10: plaque assays performed using media collected on wells from Lane 3 and 8 , respectively. 

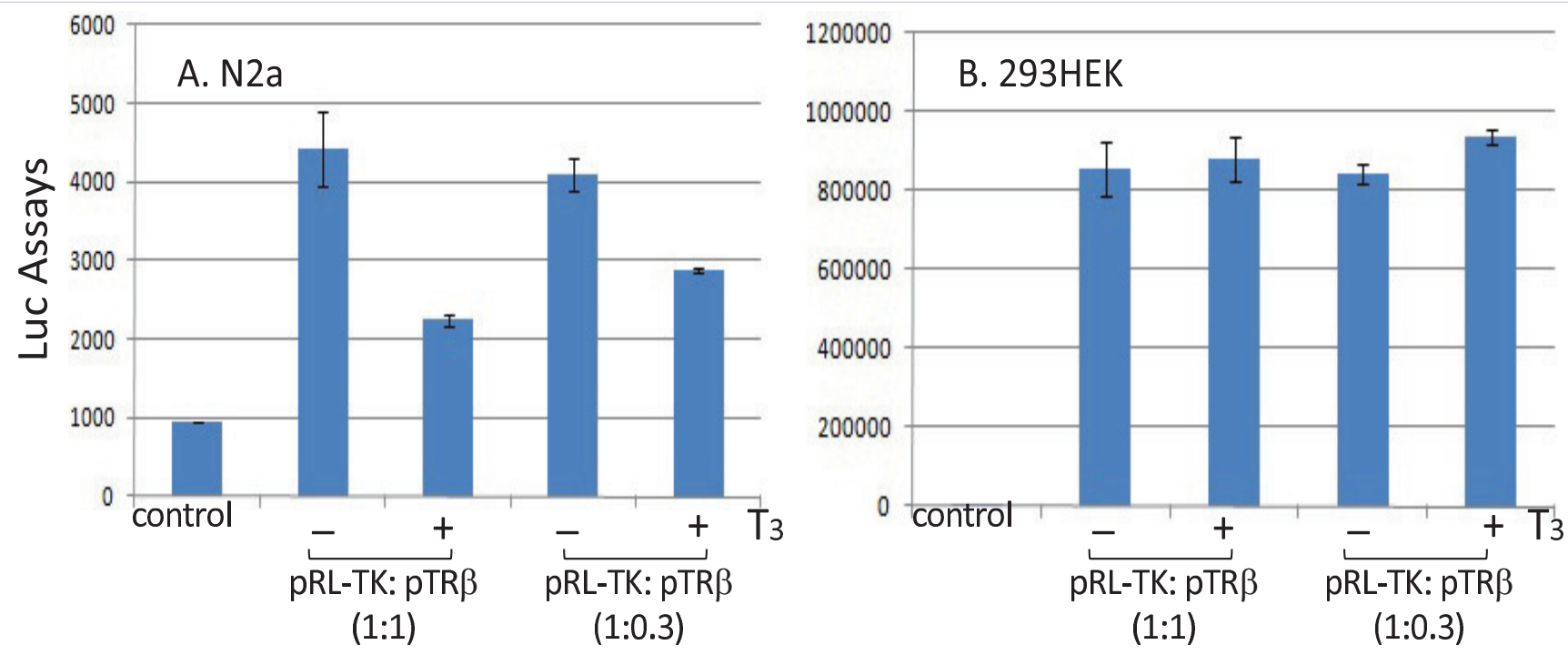

$p<0.05$

Figure 2: TR/T3 repressed TK in mouse neuronal cells

A. Co- transfection of pTR $\beta$ and pRL-TK in mouse N2a cell line regulated TK expression in presence of T3.

B. HEK 293 cells co- transfection of pTR $\beta$ and pRL-TK with or without T3 showed no regulation of TK activity from Luciferase assay.

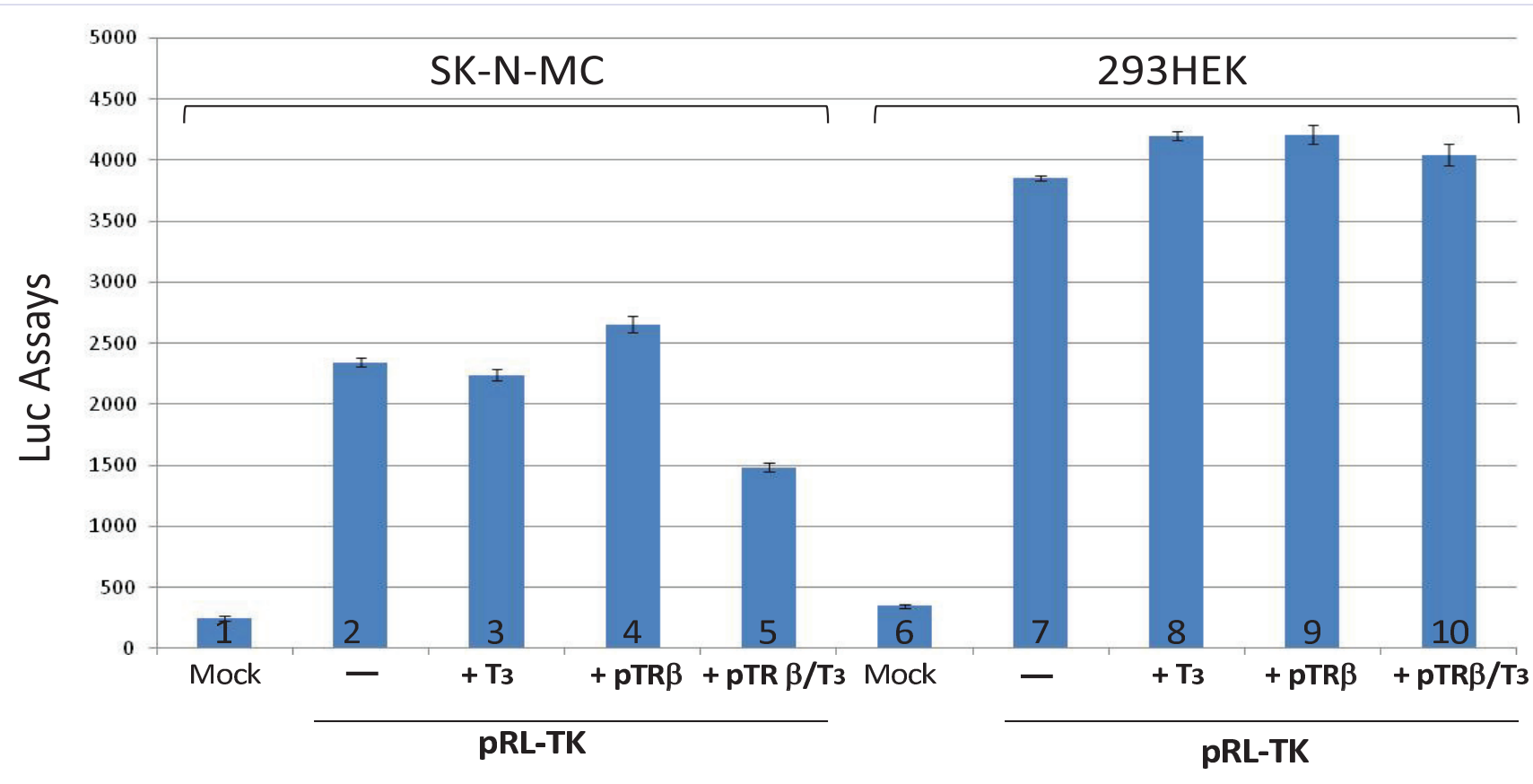

$p<0.05$

Figure 3: Human neuronal cells exhibited TK regulation by TR/T3

Transient co-transfection of pRL-TK and pTR $\beta$ in human neuronal cells SK-N-MC produced an inhibitory effect on TK promoter activity in the presence of T3. This regulation was not seen in HEK293 cells by Luciferase Assay. 
Hence, we proposed that TK regulation by T3/TR played a vital role during viral reactivation and $\mathrm{T} 3$ level was crucial to control global HSV-1 gene expression and regulate viral latency.

\section{Participation of chromosomal condensation in the T3- mediated regulation on $\mathrm{HSV}-1$ replication}

It is known that HSV-1 replicated poorly in the absence of RCC1 since temperature-sensitive cells lacking RCC1 blocked HSV-
1 replication [35-36]. Rcc1 is a signaling molecule responsible for the regulation of chromosomal condensation, sensing nonreplicating DNA, and generating the inhibitory signal to halt mitosis. Loss of RCC1 function causes premature/inappropriate chromatin condensation, and thus inhibits gene expression [37]. Therefore we investigated the role of RCC1 using our in vitro N2a cell culture model by immuno-fluorescent assays. The results showed that RCC1 coexists with cells that were infected, cells
A)

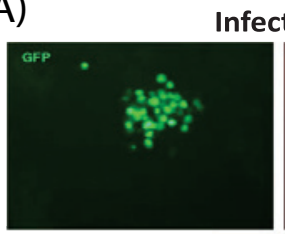

Infection
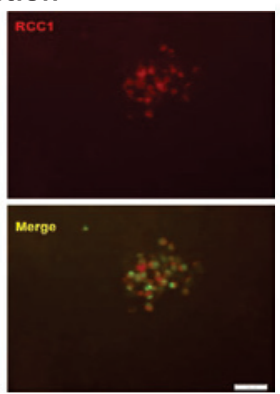
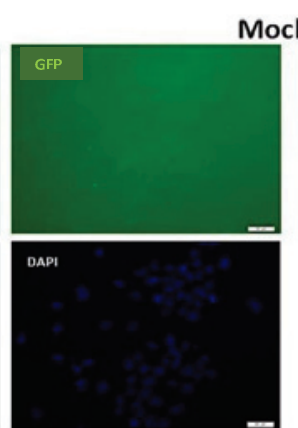

B) N2aTR
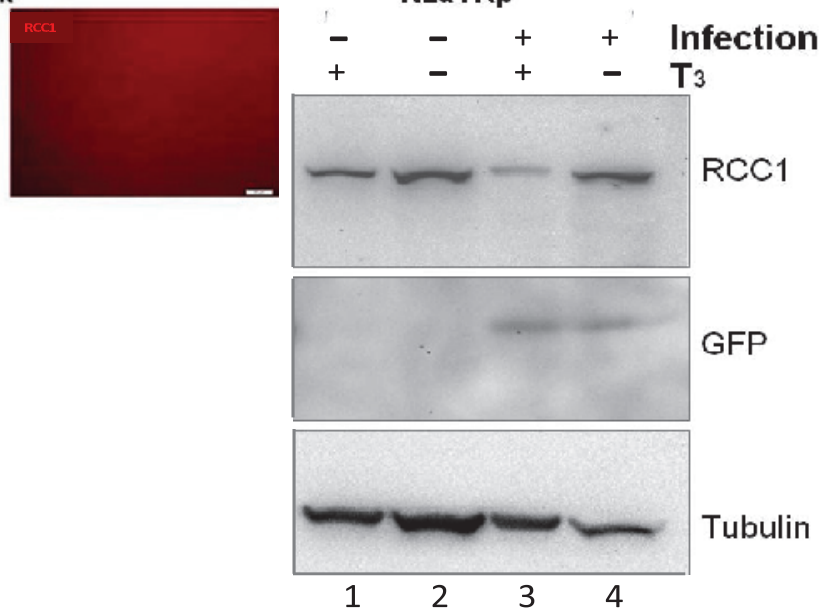

Figure 4: TR/T3 modulated RCC1 expression profile during infection

A. RCC1 was detected in N2aTR $\beta$ cells upon infection. Scale bar: $50 \mu \mathrm{m}$.

B. Western blot analyses showed that RCC1 was present in N2aTR $\beta$ cells without infection. TR/T3 decreased the RCC1 detection upon infection.

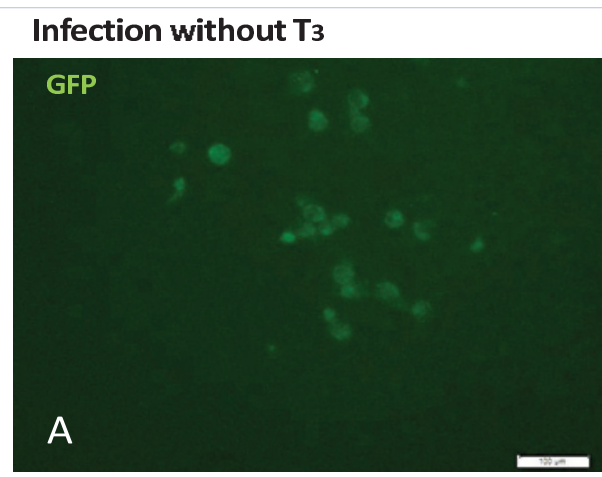

\section{Infection with T3}
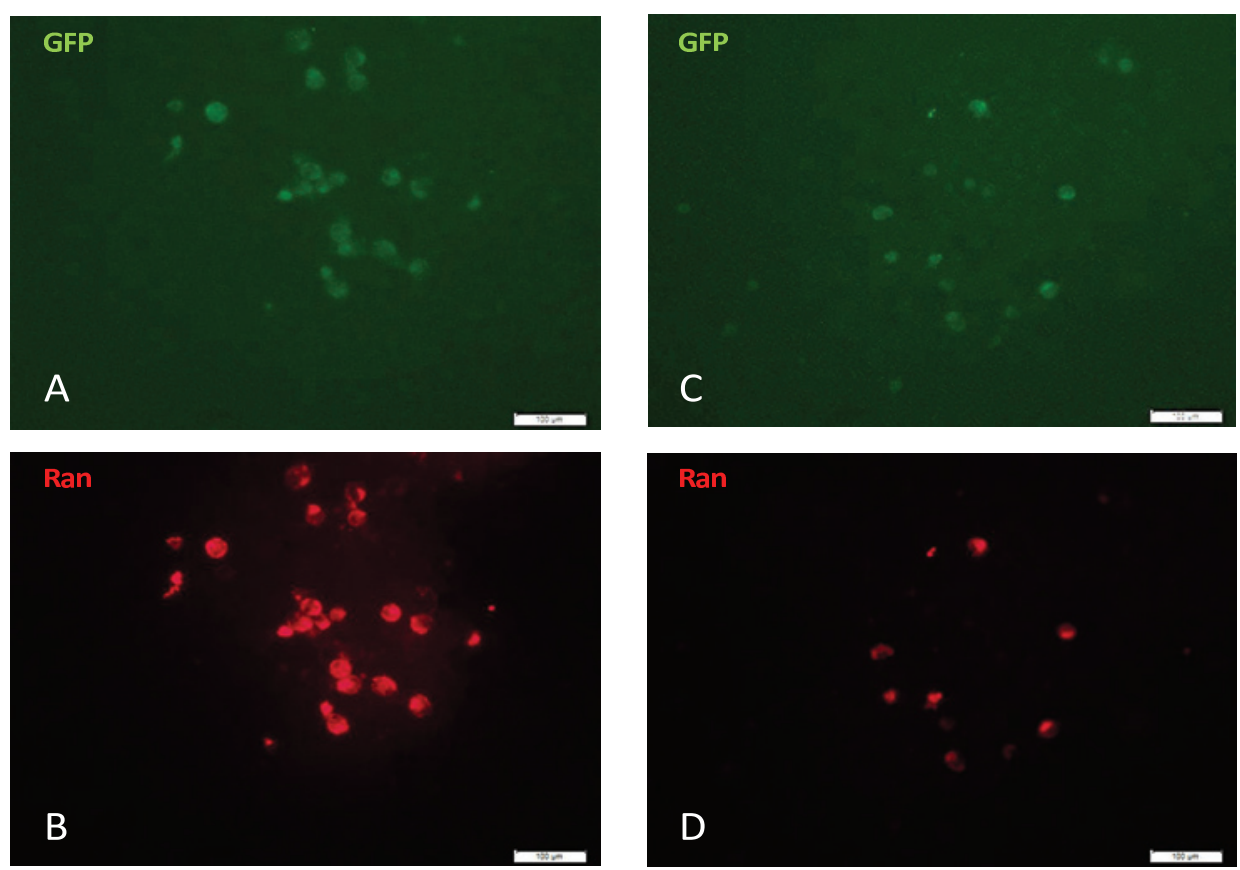

Figure 5: Ran expression was diminished in N2aTR $\beta$ cells upon infection by TR/T3. Infection of N2aTR $\beta$ cells with T3 exhibited weaker Ran expression. Bar: $100 \mu \mathrm{m}$. 
without infection exhibited no RCC1 signal (Figure 4A). However, Western blot analyses indicated that RCC1 was present in cells regardless of the status of infection but $\mathrm{T} 3$ treatment reduced the level of RCC1 (Figure 4B). The level of RCC1 was not affected when the virus infected Vero cells but was drastically reduced during infection of N2a cells in the presence of TR/T3 (data not shown). It is not understood why RCC1 was not visible in the immunofluorescent assays. The possible explanation is that RCC1 is a nuclear protein which may not be efficiently assessable during immuno-fluorescent assays, but the infection disrupted the sub cellular organization and consequently made RCC1 available. It is also likely that RCC1 was sequestered within the cellular compartment and HSV-1 infection induced post-translational modification on the RCC1 and exposed the RCC1.

In addition, RCC1 functions as a Guanine nucleotide exchange factor to control the trafficking of the nuclear $\mathrm{G}$ protein Ran (RAsrelated Nuclear protein). Ran is required for the translocation of cellular cargo such as RNA and proteins through the nuclear pore complex and also participated in the regulation of DNA synthesis and cell cycle progression [38]. Based on this information, it is likely that TR/T3 influenced the chromatin structure of HSV-1 in neuronal cells by affecting the level of RCC1/Ran complex and thus controlled mitosis, DNA replication, and cargo trafficking between nucleus and cytoplasm. To further study this novel hypothesis, we examine the Ran status in our model in the presence or absence of T3. The results of immuno-fluorescent assays indicated that when the $\mathrm{T} 3$ was removed and viral replication was active, the infected cells emitted green (virus) and red (Ran) fluorescence with strong intensity (Figure 5A and 5B). Nonetheless while T3 was available and the viral replication was repressed, the infected cells were decreased with weaker Ran signal (Figure 5C and 5D). Together these observations suggested that RCC1/Ran is beneficial to viral replication, promotes lytic infection of virus, and halts host replication. TR/ T3 may repress viral gene expression/replication via reduction of RCC1/Ran expression/availability and thus keep the viral DNA in circular and promote latency.

In conclusion, our results showed that thyroid hormone exerts a novel negative regulation on HSV-1 gene TK and it required TR and neuronal environment. More studies are underway to investigate the roles of chromatin condensation, histone modification, chromatin remodeling, chromatin insulation in the TR/T3 mediated HSV-1 gene regulation and replication in neuronal environment.

\section{Acknowledgment}

We thank Dr. Timothy Foster of LSUHSC in New Orleans and Dr. Gus Kousoulas from LSU Baton Rouge, LA for the $17 \mathrm{Syn}^{+}-$ EGFP virus. Our gratitude also goes to Dr. Robert Denver from University of Michigan in Ann Arbor, MI for the N2aTR $\beta$ cell line. We are grateful for the assistance of the editorial staff at University of Maryland Eastern Shore. This project is supported by NIH R01NS081109 to SVH. The content is solely the responsibility of the authors and does not necessarily represent the official views of the NINDS/NIH.

\section{References}

1. Lopez Garcia FR, Enriquez Ascarza R, Rodriguez Martinez JC, Sirvent Pedreno AE (2002) Interferon therapy for herpes simplex virus infection in a 70 years old patient. An Med Interna 19: 600-601.

2. Taylor TJ, Brockman MA, McNamee EE, Knipe DM (2002) Herpes simplex virus. Front Biosci 7: 752-764.

3. Everett RD (2000) ICP0, a regulator of herpes simplex virus during lytic and latent infection. Bioessays 22: 761-770.

4. Shah A, Farooq AV, Tiwari V, Kim MJ, Shukla D (2010) HSV-1 infection of human corneal epithelial cells: receptor-mediated entry and trends of re-infection. Mol Vis 16: 2476-2486.

5. Usatine RP, R Tinitigan (2010) Nongenital herpes simplex virus. Am Fam Physician 82: 1075-1082.

6. Higaki S, Y Inoue, Yoshida A, Maeda N, Watanabe H, et al. (2008) Case of bilateral multiple herpetic epithelial keratitis manifested as dendriform epithelial edema during primary Kaposi's varicelliform eruption. Jpn J Ophthalmol 52: 127-129.

7. Martinez PA, R Diaz, Gonzalez D, Oropesa L, Gonzalez R, et al. (2007) The effect of highly active antiretroviral therapy on outcome of central nervous system herpesviruses infection in Cuban human immunodeficiency virus-infected individuals. J Neurovirol 13: 446451.

8. Goel N, H Mao, Rong Q, Docherty JJ, Zimmerman D, et al. (2002) The ability of an HSV strain to initiate zosteriform spread correlates with its neuroinvasive disease potential. Arch Virol 147: 763-773.

9. Hsia, SC, Bedadala GR, Balish MD (2011) Effects of thyroid hormone on HSV-1 gene regulation: implications in the control of viral latency and reactivation. Cell Biosci 1: 1-24.

10. Garza HH Jr, Hill JM (1997) Effect of a beta-adrenergic antagonist, propranolol, on induced HSV-1 ocular recurrence in latently infected rabbits. Curr Eye Res 16: 453-458.

11. Hardwicke MA, PA Schaffer (1997) Differential effects of nerve growth factor and dexamethasone on herpes simplex virus type 1 oriL- and oriS-dependent DNA replication in PC12 cells. J Virol 71: 3580-3587.

12. Noisakran S, Halford Veress WP, Veress L, Carr DJ (1998) Role of the hypothalamic pituitary adrenal axis and IL-6 in stress-induced reactivation of latent herpes simplex virus type 1 . J Immunol 160 : 5441-5447.

13. Marquart M, Bhattacharjee $P$, Zheng $X$, Kaufman $H$, Thompson $H$, et al. (2003) Ocular reactivation phenotype of HSV-1 strain F(MP)E, a corticosteroid-sensitive strain. Curr Eye Res 26: 205-209.

14. Bedadala GR, Pinnoji RC, Palem JR, Hsia SC (2010) Thyroid hormone controls the gene expression of HSV-1 LAT and ICP0 in neuronal cells. Cell Res 20: 587-598.

15. Hsia SC, Pinnoji RC, Bedadala GR, Hill JM, Palem JR (2010) Regulation of herpes simplex virus type 1 thymidine kinase gene expression by thyroid hormone receptor in cultured neuronal cells. J Neurovirol 16: 13-24.

16.16. Varedi M, Moattari A, Amirghofran Z, Karamizadeh Z, Feizi H (2013) Effects of hypo- and hyperthyroid states on herpes simplex virus infectivity in the rat. Endocr Res 39: 50-55.

17. Deme D, J Pommier, Nunez J (1976) Kinetics of thyroglobulin iodination and of hormone synthesis catalysed by thyroid peroxidase. Role of iodide in the coupling reaction. Eur J Biochem 70: 435-440. 
18. Tsai MJ, O'Malley BW (1994) Molecular mechanisms of action of steroid/thyroid receptor superfamily members. Annu Rev Biochem 63: 451-486.

19. Flamant F, Baxter JD, Forrest D, Refetoff S, Samuels H, et al. (2006) International Union of Pharmacology. LIX. The pharmacology and classification of the nuclear receptor superfamily: thyroid hormone receptors. Pharmacol Rev 58: 705-711.

20. Kubat NJ, Amelio AL, Giordani NV, Bloom DC (2004) The herpes simplex virus type 1 latency-associated transcript (LAT) enhancer/rcr is hyperacetylated during latency independently of LAT transcription. J Virol 78: 12508-12518.

21. Kubat NJ, Tran RK, McAnany P, Bloom DC (2004) Specific histone tail modification and not DNA methylation is a determinant of herpes simplex virus type 1 latent gene expression. J Virol 78: 1139-1149.

22. Amelio AL, McAnany PK, Bloom DC et al (2006) A chromatin insulatorlike element in the herpes simplex virus type 1 latency-associated transcript region binds CCCTC-binding factor and displays enhancerblocking and silencing activities. J Virol 80: 2358-2368.

23. Bedadala GR, RC Pinnoji, Hsia SC (2007)Early growth response gene 1 (Egr-1) regulates HSV-1 ICP4 and ICP22 gene expression. Cell Res 17: 546-555.

24. Chen Q, Lin L, Sheryl Smith, Jing Huang, Shelley L Berger, et al. (2007) A CTCF-dependent chromatin boundary element exists between the LAT and ICP0 promoters in the HSV-1 genome. J Virol 10: 5192-5201.

25. Pinnoji RC, Bedadala GR, George B, Holland TC, Hill JM, et al. (2007) Repressor element-1 silencing transcription factor/neuronal restrictive silencer factor (REST/NRSF) can regulate HSV-1 immediate-early transcription via histone modification. Virol J 4: 56.

26. Knipe DM, Cliffe A (2008) Chromatin control of herpes simplex virus lytic and latent infection. Nat Rev Microbiol 6: 211-221.

27. Foster TP, Rybachuk GV, Kousoulas KG (1998) Expression of the enhanced green fluorescent protein by herpes simplex virus type 1 (HSV-1) as an in vitro or in vivo marker for virus entry and replication. J Virol Methods 75: 151-160.

28. Bedadala GR, Palem JR, Graham L, Hill JM, McFerrin HE, et al. (2011)
Lytic HSV-1 infection induces the multifunctional transcription factor Early Growth Response-1 (EGR-1) in rabbit corneal cells. Virol J 8: 262.

29. Shaw G, Morse S, Ararat M, Graham FL (2002)Preferential transformation of human neuronal cells by human adenoviruses and the origin of HEK 293 cells. FASEB J 16: 869-871.

30. Lebel JM, Dussault JH, Puymirat J (1994) Overexpression of the beta 1 thyroid receptor induces differentiation in neuro-2a cells. Proc Natl Acad Sci U S A 91: 2644-2648.

31. Nichol PF, Chang JY, Johnson EM Jr, Olivo PD (1996) Herpes simplex virus gene expression in neurons: viral DNA synthesis is a critical regulatory event in the branch point between the lytic and latent pathways. J Virol 70: 5476-5486.

32. Tal-Singer R, Peng C, Ponce De Leon M, Abrams WR, Banfield BW, et al. (1995). Interaction of herpes simplex virus glycoprotein gC with mammalian cell surface molecules. J Virol 69: 4471-4483.

33. Tal-Singer R, Lasner TM, Podrzucki W, Skokotas A, Leary JJ, et al. (1997). Gene expression during reactivation of herpes simplex virus type 1 from latency in the peripheral nervous system is different from that during lytic infection of tissue cultures. J Virol 71: 5268-5276.

34. Kosz-Vnenchak M, Jacobson J, Coen DM, Knipe DM (1993) Evidence for a novel regulatory pathway for herpes simplex virus gene expression in trigeminal ganglion neurons. J Virol 67: 5383-5393.

35. Umene K, Nishimoto T (1996) Replication of herpes simplex virus type 1 DNA is inhibited in a temperature-sensitive mutant of BHK-21 cells lacking RCC1 (regulator of chromosome condensation) and virus DNA remains linear. J Gen Virol 77: 2261-2270.

36. Strang BL, Stow ND (2007) Blocks to herpes simplex virus type 1 replication in a cell line, tsBN2, encoding a temperature-sensitive RCC1 protein. J Gen Virol 88: 376-383.

37. Dasso M (1993) RCC1 in the cell cycle: the regulator of chromosome condensation takes on new roles. Trends Biochem Sci 18: 96-101.

38. Pemberton LF, Paschal BM (2005) Mechanisms of receptor-mediated nuclear import and nuclear export. Traffic 6: 187-198. 